\title{
OPTIMIZATION AND CONVERGENCE OF OBSERVATION CHANNELS IN STOCHASTIC CONTROL
}

\author{
SERDAR YÜKSEL AND TAMÁS LINDER
}

\begin{abstract}
This paper studies the optimization of observation channels (stochastic kernels) in partially observed stochastic control problems. In particular, existence and continuity properties are investigated mostly (but not exclusively) concentrating on the single-stage case. Continuity properties of the optimal cost in channels are explored under total variation, setwise convergence, and weak convergence. Sufficient conditions for compactness of a class of channels under total variation and setwise convergence are presented and applications to quantization are explored.
\end{abstract}

Key words. Stochastic control, information theory, observation channels, optimization, quantization

AMS subject classifications. 15A15, 15A09, 15A23

1. Introduction. In stochastic control, one is often concerned with the following problem: Given a dynamical system, an observation channel (stochastic kernel), a cost function, and an action set, when does there exist an optimal policy, and what is an optimal control policy? The theory for such problems is advanced, and practically significant, spanning a wide variety of applications in engineering, economics, and natural sciences.

In this paper, we are interested in a dual problem with the following questions to be explored: Given a dynamical system, a cost function, an action set, and a set of observation channels, does there exist an optimal observation channel? What is the right convergence notion for continuity in such observation channels for optimization purposes? The answers to these questions may provide useful tools for characterizing an optimal observation channel subject to constraints.

We start with the probabilistic setup of the problem. Let $\mathbb{X} \subset \mathbb{R}^{n}$, be a Borel set in which elements of a controlled Markov process $\left\{X_{t}, t \in \mathbb{Z}_{+}\right\}$live. Here and throughout the paper $\mathbb{Z}_{+}$denotes the set of nonnegative integers and $\mathbb{N}$ denotes the set of positive integers. Let $\mathbb{Y} \subset \mathbb{R}^{m}$ be a Borel set, and let an observation channel $Q$ be defined as a stochastic kernel (regular conditional probability) from $\mathbb{X}$ to $\mathbb{Y}$, such that $Q(\cdot \mid x)$ is a probability measure on the (Borel) $\sigma$-algebra $\mathcal{B}(\mathbb{Y})$ on $\mathbb{Y}$ for every $x \in \mathbb{X}$, and $Q(A \mid \cdot): \mathbb{X} \rightarrow[0,1]$ is a Borel measurable function for every $A \in \mathcal{B}(\mathbb{Y})$. Let a decision maker (DM) be located at the output an observation channel $Q$, with inputs $X_{t}$ and outputs $Y_{t}$. Let $\mathbb{U}$ be a Borel subset of some Euclidean space. An admissible policy $\Pi$ is a sequence of control functions $\left\{\gamma_{t}, t \in \mathbb{Z}_{+}\right\}$such that $\gamma_{t}$ is measurable with respect to the $\sigma$-algebra generated by the information variables

$$
I_{t}=\left\{Y_{[0, t]}, U_{[0, t-1]}\right\}, \quad t \in \mathbb{N}, \quad I_{0}=\left\{Y_{0}\right\}
$$

where

$$
U_{t}=\gamma_{t}\left(I_{t}\right), \quad t \in \mathbb{Z}_{+}
$$

The material of this paper was presented in part at the 2010 Information Theory and Applications Workshop, University of California, San Diego, Feb. 2010.

The authors are with the Department of Mathematics and Statistics, Queen's University, Kingston, Ontario, Canada, K7L 3N6. Email: (yuksel,linder)@mast.queensu.ca. This research was partially supported by the Natural Sciences and Engineering Research Council of Canada (NSERC). 
are the $\mathbb{U}$-valued control actions and we used the notation

$$
Y_{[0, t]}=\left\{Y_{s}, 0 \leq s \leq t\right\}, \quad U_{[0, t-1]}=\left\{U_{s}, 0 \leq s \leq t-1\right\} .
$$

The joint distribution of the state, control, and observation processes is determined by (1.1) and the following relationships:

$$
\operatorname{Pr}\left(\left(X_{0}, Y_{0}\right) \in B\right)=\int_{B} P\left(d x_{0}\right) Q\left(d y_{0} \mid x_{0}\right), \quad B \in \mathcal{B}(\mathbb{X} \times \mathbb{Y}),
$$

where $P$ is the (prior) distribution of the initial state $X_{0}$, and

$$
\begin{aligned}
& \operatorname{Pr}\left(\left(X_{t}, Y_{t}\right) \in B \mid X_{[0, t-1]}=x_{[0, t-1]}, Y_{[0, t-1]}=y_{[0, t-1]}, U_{[0, t-1]}=u_{[0, t-1]}\right) \\
&=\int_{B} P\left(d x_{t} \mid x_{t-1}, u_{t-1}\right) Q\left(d y_{t} \mid x_{t}\right), \quad B \in \mathcal{B}(\mathbb{X} \times \mathbb{Y}), \quad t \in \mathbb{N},
\end{aligned}
$$

where $P(\cdot \mid x, u)$ is a stochastic kernel from $\mathbb{X} \times \mathbb{U}$ to $\mathbb{X}$.

One way of presenting the problem in a familiar setting is the following: Consider a dynamical system described by the discrete-time equations

$$
\begin{aligned}
X_{t+1} & =f\left(X_{t}, U_{t}, W_{t}\right), \\
Y_{t} & =g\left(X_{t}, V_{t}\right)
\end{aligned}
$$

for some measurable functions $f, g$, with $\left\{W_{t}\right\}$ being independent and identically distributed (i.i.d) system noise process and $\left\{V_{t}\right\}$ an i.i.d. disturbance process, which are independent of $X_{0}$ and each other. Here, the second equation represents the communication channel $Q$, as it describes the relation between the state and observation variables.

With the above setup, let the objective of the decision maker be the minimization of the cost

$$
J(P, Q, \Pi)=E_{P}^{Q, \Pi}\left[\sum_{t=0}^{T-1} c\left(X_{t}, U_{t}\right)\right],
$$

over the set of all admissible policies $\Pi$, where $c: \mathbb{X} \times \mathbb{U} \rightarrow \mathbb{R}$ is a Borel measurable cost function and $E_{P}^{Q, \Pi}$ denotes the expectation with initial state probability measure given by $P$ under policy $\Pi$ and given channel $Q$. We adapt the convention that random variables are denoted by capital letters and lowercase letters denote their realizations. Also, given a probability measure $\mu$ the notation $Z \sim \mu$ means that $Z$ is a random variable with distribution $\mu$. Finally, let $\mathscr{P}$ be the set of all admissible policies $\Pi$ described above.

We are interested in the following problems:

Problem P1. Continuity on the SPaCe of Channels (Stochastic Kernels) Suppose $\left\{Q_{n}, n \in \mathbb{N}\right\}$ is a sequence of communication channels converging in some sense to a channel $Q$. When does

$$
Q_{n} \rightarrow Q
$$

imply

$$
\inf _{\Pi \in \mathscr{P}} J\left(P, Q_{n}, \Pi\right) \rightarrow \inf _{\Pi \in \mathscr{P}} J(P, Q, \Pi) ?
$$


Problem P2: Existence of optimal Channels Let $\mathcal{Q}$ be a set of communication channels. When do there exist minimizing and maximizing channels for the problems

$$
\inf _{Q \in \mathcal{Q}} \inf _{\Pi \in \mathscr{P}} E_{P}^{Q, \Pi}\left[\sum_{t=0}^{T-1} c\left(X_{t}, U_{t}\right)\right]
$$

and

$$
\sup _{Q \in \mathcal{Q}} \inf _{\Pi \in \mathscr{P}} E_{P}^{Q, \Pi}\left[\sum_{t=0}^{T-1} c\left(X_{t}, U_{t}\right)\right] .
$$

If solutions to these problems exist, are they unique?

Problems P1 and P2 are challenging even in the single-stage $(T=1)$ setup and in most of the paper we consider this case. Admittedly, the multi-stage case is more important and we briefly consider this case is Section 6 at the end of the paper. Future work is needed to fully address this technically more complex case.

The answers to problems P1 and P2 may help solve problems in application areas such as:

- For a partially observed stochastic control problem, sometimes we have control over the observation channels by encoding/quantization. When does there exist an optimal quantizer for such a setup? (Optimal quantization)

- Given an uncertainty set for the observation channels, can one identify a worst element/best element? (Robust control)

- When estimating channels from empirical observations, under quite general assumptions estimations converge to the actual distribution, in some sense. For example, if an observation channel has the form $Y_{t}=X_{t}+V_{t}$, where the independent noise $V_{t}$ has a density, nonparametric density estimation methods lead to convergence in total variation, whereas for the general case, the empirical measures converge weakly with probability one [10, [13. Do these modes of convergence imply that we could design the optimal control policies based on empirical estimates, and does the optimal cost converge to the correct limit as the number of measurements grows? (Consistency of empirical controllers)

In the following, we will address problems $\mathrm{P} 1$ and P2 and introduce conditions under which we can provide affirmative/conclusive answers.

1.1. Relevant literature. The problems stated are related to three main areas of research: Robust control, optimal quantizer design and design of experiments.

References [8, 26, 28, have considered both optimal control and estimation and the related problem of optimal control design when the channel is unknown. In particular, 28. studies the existence of optimal continuous estimation policies and worst-case channels under a relative entropy constraint characterizing the uncertainty in the system. In [26], the total variation norm is considered as the measure of the uncertainty, and the inf-sup policy is determined (thus, the setup considered as a min-max problem for the generation of optimal control policies). Similarly, there are connections with robust detection, such as those studied by Huber [21] and Poor [25], when the source distribution to be detected belongs to some set.

A related area is on the theory of optimal quantization: References [1, [16] are related as these papers study the effects of uncertainties in the input distribution and consider robustness in the quantizer design. References [22] and 24] study the 
consistency of optimal quantizers based on empirical data for an unknown source. In the context of decentralized detection, 29] studied certain topological properties and the existence of optimal quantizers. We will regard the quantizers as a particular class of channels, and look for such optimal channels. One by-product of our analysis will be a new approach to obtain conditions for the existence of optimal quantizers for a given class of cost functions under mild conditions. We also note that, regarding connections with information theory, some discussions on the topology of information channels are presented in 23]. Recently, 34] considered continuity and other functional properties of minimum mean square estimation problems under Gaussian channels.

As mentioned earlier, in most of the paper we consider the single-stage case. We will also briefly consider the technically more complex multi-stage case in Section 6 where further conditions on the controlled Markov chain must be imposed. The full development of this general setup is the subject of future work.

The rest of the paper is organized as follows. In the next section, we introduce three relevant topologies on the space of communication channels. The continuity problem is considered in Section 3. We study the problem of existence of optimal channels in Section 4, followed by applications on quantization in Section 5ection 6 gives an outlook to the multi-stage setup. The paper ends with the concluding remarks and discussions in Section 7.

2. Some topologies on the space of communication channels. One question that we wish address is the choice of an appropriate notion of convergence for a sequence of observation channels. Toward this end, we first review three notions of convergence for probability measures.

Let $\mathcal{P}\left(\mathbb{R}^{\mathbb{N}}\right)$ denote the family of all probability measure on $\left(\mathbb{X}, \mathcal{B}\left(\mathbb{R}^{N}\right)\right)$ for some $N \in \mathbb{N}$. Let $\left\{\mu_{n}, n \in \mathbb{N}\right\}$ be a sequence in $\mathcal{P}\left(\mathbb{R}^{N}\right)$. Recall that $\left\{\mu_{n}\right\}$ is said to converge to $\mu \in \mathcal{P}\left(\mathbb{R}^{N}\right)$ weakly if

$$
\int_{\mathbb{R}^{N}} c(x) \mu_{n}(d x) \rightarrow \int_{\mathbb{R}^{N}} c(x) \mu(d x)
$$

for every continuous and bounded $c: \mathbb{R}^{N} \rightarrow \mathbb{R}$. On the other hand, $\left\{\mu_{n}\right\}$ is said to converge to $\mu \in \mathcal{P}\left(\mathbb{R}^{N}\right)$ setwise if

$$
\int_{\mathbb{R}^{N}} c(x) \mu_{n}(d x) \rightarrow \int_{\mathbb{R}^{N}} c(x) \mu(d x)
$$

for every measurable and bounded $c: \mathbb{R}^{N} \rightarrow \mathbb{R}$. Setwise convergence can also be defined through pointwise convergence on Borel subsets of $\mathbb{R}^{N}$ (see, e.g., [20]), that is

$$
\mu_{n}(A) \rightarrow \mu(A), \quad \text { for all } A \in \mathcal{B}\left(\mathbb{R}^{N}\right)
$$

since the space of simple functions is dense in the space of bounded and measurable functions under the supremum norm.

For two probability measures $\mu, \nu \in \mathcal{P}\left(\mathbb{R}^{N}\right)$, the total variation metric is given by

$$
\begin{aligned}
\|\mu-\nu\|_{T V} & :=2 \sup _{B \in \mathcal{B}\left(\mathbb{R}^{N}\right)}|\mu(B)-\nu(B)| \\
& =\sup _{f:\|f\|_{\infty} \leq 1}\left|\int f(x) \mu(d x)-\int f(x) \nu(d x)\right|,
\end{aligned}
$$

where the supremum is over all measurable real $f$ such that $\|f\|_{\infty}=\sup _{x \in \mathbb{R}^{N}}|f(x)| \leq$ 1. A sequence $\left\{\mu_{n}\right\}$ is said to converge to $\mu \in \mathcal{P}\left(\mathbb{R}^{N}\right)$ in total variation if $\left\|\mu_{n}-\mu\right\|_{T V} \rightarrow$ 0 . 
Setwise convergence is equivalent to pointwise convergence on Borel sets whereas convergence in total variation requires uniform convergence on Borel sets. Thus convergence in total variation implies setwise convergence, which in turn implies weak convergence. It follows that the induced topologies are of decreasing order of strength, with the topology induced by convergence in total variation being the strongest and the topology induced by weak convergence being the weakest, with the topology induced by setwise convergence is in between these two. The topologies corresponding to convergence in total variation and weak convergence are metrizable (the natural metric for total variation convergence is $d(\mu, \nu)=\|\nu-\nu\|_{T V}$; the usual choice for weak convergence is the Prohorov metric [4]). The topology induced by setwise convergence is not first countable, so it is not metrizable (see, e.g., [14, Prop. 2.2.1]).

2.1. Convergence of information (observation) channels. Here $\mathbb{X}=\mathbb{R}^{n}$ and $\mathbb{Y}=\mathbb{R}^{m}$, and $\mathcal{Q}$ denotes the set of all observation channels (stochastic kernels) with input space $\mathbb{X}$ and output space $\mathbb{Y}$. For $P \in \mathcal{P}(\mathbb{X})$ and $Q \in \mathcal{Q}$ we let $P Q$ denote the joint distribution induced on $(\mathbb{X} \times \mathbb{Y}, \mathcal{B}(\mathbb{X} \times \mathbb{Y}))$ by channel $Q$ with input distribution $P$ :

$$
P Q(A)=\int_{A} Q(d y \mid x) P(d x), \quad A \in \mathcal{B}(\mathbb{X} \times \mathbb{Y})
$$

Definition 2.1 (Convergence of Channels).

(i) A sequence of channels $\left\{Q_{n}\right\}$ converges to a channel $Q$ weakly at input $P$ if $P Q_{n} \rightarrow P Q$ weakly.

(ii) A sequence of channels $\left\{Q_{n}\right\}$ converges to a channel $Q$ setwise at input $P$ if $P Q_{n} \rightarrow P Q$ setwise, i.e., if $P Q_{n}(A) \rightarrow P Q(A)$ for all Borel sets $A \subset \mathbb{X} \times \mathbb{Y}$.

(iii) A sequence of channels $\left\{Q_{n}\right\}$ converges to a channel $Q$ in total variation at input $P$ if $P Q_{n} \rightarrow P Q$ in total variation, i.e., if $\left\|P Q_{n}-P Q\right\|_{T V} \rightarrow 0$.

If we introduce the equivalence relation $Q \stackrel{P}{\equiv} Q^{\prime}$ if and only if $P Q=P Q^{\prime}$, $Q, Q^{\prime} \in \mathcal{Q}$, then the convergence notions in Definition 2.1 only induce the corresponding topologies (resp. metrics) on the resulting equivalence classes in $\mathcal{Q}$, instead of $\mathcal{Q}$. Since in most of the development the input distribution $P$ is fixed, there should be no confusion when (somewhat incorrectly) we talk about the induced topologies (resp. metrics) on $\mathcal{Q}$.

The preceding definition involved the input distribution $P$. The next lemma gives sufficient conditions which may be easier to verify. The proof is given in the Appendix.

LEMMA 2.2. weakly.

(i) If $\left\{Q_{n}(\cdot \mid x)\right\}$ converges to $Q(\cdot \mid x)$ weakly for $P$-a.e. $x$, then $P Q_{n} \rightarrow P Q$ setwise.

(ii) If $\left\{Q_{n}(\cdot \mid x)\right\}$ converges to $Q(\cdot \mid x)$ setwise for $P$-a.e. $x$, then $P Q_{n} \rightarrow P Q$

(iii) If $\left\{Q_{n}(\cdot \mid x)\right\}$ converges to $Q(\cdot \mid x)$ in total variation for $P$-a.e. $x$, then $P Q_{n} \rightarrow$ $P Q$ in total variation.

The conditions in Lemma 2.2 are almost universal in the choice of input probability measures; that is, the convergence characterizations will be independent of the input distributions if each of the conditions is replaced with convergence of $\left\{Q_{n}(\cdot \mid x)\right\}$ to $Q(\cdot \mid x)$ for all $x \in \mathbb{X}$. This is particularly useful when the input distribution is unknown, or when the input distributions may change. The latter can occur in multi-stage stochastic control problems. 
EXAMPLE 2.3.

(i) Consider the case where the observation channel has the form $Y_{t}=X_{t}+V_{t}$, where $\left\{V_{t}\right\}$ is an i.i.d. noise (disturbance) process. Suppose $V_{t} \sim f_{\theta_{0}}$ for some $\theta_{0} \in \Theta$, where $\Theta \subset \mathbb{R}^{d}$ is a parameter set and $\left\{f_{\theta}: \theta \in \Theta\right\}$ is a parametric family of $n$ dimensional densities such that $f_{\theta_{n}}(v) \rightarrow f_{\theta_{0}}(v)$ for all $v \in \mathbb{R}^{n}$ and any sequence of parameters $\theta_{n}$ such that $\theta_{n} \rightarrow \theta_{0}$. Then by Scheffé's theorem $f_{\theta_{n}}$ converges to $f_{\theta_{0}}$ in the $L_{1}$ sense, and consequently, the sequence of corresponding additive channels $Q_{n}(\cdot \mid x)$, defined by

$$
Q_{n}(A \mid x)=\int_{A} f_{\theta_{n}}(z-x) d z, \quad A \in \mathcal{B}\left(\mathbb{R}^{n}\right)
$$

converges to the channel $Q(\cdot \mid x)$ (corresponding to $f_{\theta}$ ) in total variation for all $x$.

(ii) Consider again the observation channel $Y_{t}=X_{t}+V_{t}$, but assume this time that we only know that $V_{t}$ has a density $f$ (which is unknown to us). If we have access to independent observations $V_{1}, \ldots, V_{n}$ from the noise process, then we can use any of the consistent nonparametric methods, e.g., [10, to obtain an estimate $f_{n}$ which converges (with probability one) to $f$ in the $L_{1}$ sense as $n \rightarrow \infty$. More explicitly, letting $(\Omega, \mathcal{A}, \mathbb{P})$ be the probability space on which the independent observations $\left\{V_{i}\right\}$ are defined, for any $\omega \in \Omega$, the estimate $f_{n}=f_{n, \omega}$ is a pdf on $\mathbb{R}^{n}$, and there exists $A \in \mathcal{A}$ with $\mathbb{P}(A)=1$ such that $\int\left|f_{n, \omega}(z)-f_{n}(z)\right| d z \rightarrow 0$ as $n \rightarrow \infty$ for all $\omega \in A$. The estimated channel $Q_{n}(\cdot \mid x)=Q_{n, \omega}(\cdot \mid x)$ corresponding to $f_{n, \omega}$ converges to the true channel $Q(\cdot \mid x)$ in total variation for all $x$ with probability one. More explicitly, for any $\omega \in A, Q_{n, \omega}(\cdot \mid x)$ converges to $Q(\cdot \mid x)$ in total variation as $n \rightarrow \infty$ for all $x$.

(iii) Now suppose that the observation channel $Q$ is such that $Q(\cdot \mid x)$ admits a conditional density $f(y \mid x)$ for all $x \in \mathbb{R}^{n}$. Given observations $\left(X_{1}, Y_{n}\right), \ldots,\left(X_{n}, Y_{n}\right)$ drawn independently from the distribution $P Q$, there exists a sequence of nonparametric conditional density estimates $f_{n}(y \mid x)$ such that

$$
\int\left(\int\left|f_{n}(y \mid x)-f(y \mid x)\right| d y\right) P(d x) \rightarrow 0
$$

with probability one [17. This immediately implies that the channels $Q_{n}$ corresponding to these estimates converge to $Q$ in total variation at input $P$.

(iv) Finally, assume again the additive model $Y_{t}=X_{t}+V_{t}$, where now we do not have any information about the distribution $\mu$ of $V_{t}$. In this case there are no methods to consistently estimate $\mu$ in total variation from independent samples $V_{1}, \ldots, V_{n}$ [11. However, the empirical distribution $\mu_{n}$ of the samples converges weakly to $\mu$ with probability one [13]. The corresponding estimated observation channels $Q_{n}(\cdot \mid x)$ converge weakly to the true channel $Q(\cdot \mid x)$ for all $x$ with probability one.

2.2. Classes of assumptions. Throughout the paper the following classes of assumptions will be adopted for the cost function $c$ and the (Borel) set $\mathbb{U} \subset \mathbb{R}^{k}$ in different contexts:

Assumptions.

A1. The function $c: \mathbb{X} \times \mathbb{U} \rightarrow \mathbb{R}$ is non-negative, bounded, and continuous on $\mathbb{X} \times \mathbb{U}$.

A2. The function function $c: \mathbb{X} \times \mathbb{U} \rightarrow \mathbb{R}$ is non-negative, measurable, and bounded.

A3. The function $c: \mathbb{X} \times \mathbb{U} \rightarrow \mathbb{R}$ is non-negative, measurable, bounded, and continuous on $\mathbb{U}$ for every $x \in \mathbb{X}$. 
A4. $\mathbb{U}$ is a compact set.

A5. $\mathbb{U}$ is a convex set.

3. Problem P1: Continuity of the optimal cost in channels. In this section, we consider continuity properties under total variation, setwise convergence and weak convergence. We consider the single-stage case, and thus investigate the continuity of the functional

$$
\begin{aligned}
J(P, Q) & =\inf _{\Pi} E_{P}^{Q, \Pi}\left[c\left(X_{0}, U_{0}\right)\right] \\
& =\inf _{\gamma \in \mathcal{G}} \int_{\mathbb{X} \times \mathbb{Y}} c(x, \gamma(y)) Q(d y \mid x) P(d x)
\end{aligned}
$$

in the channel $Q$, where $\mathcal{G}$ is the collection of all Borel measurable functions mapping $\mathbb{Y}$ into $\mathbb{U}$. Note that by our previous notation, $\Pi=\gamma$ is an admissible first-stage control policy. As before, in this section $\mathcal{Q}$ denotes the set of all channels with input space $\mathbb{X}$ and output space $\mathbb{Y}$.

Total variation is a stringent notion for convergence. For example a sequence of discrete probability measures never converges in total variation to a probability measure which admits a density function with respect to the Lebesgue measure. On the other hand, setwise convergence induces a topology on the space of probability measures and channels which is not easy to work with. This is mainly due to the property that the space under this convergence is not metrizable. However, the space of probability measures on a complete, separable, metric (Polish) space endowed with the topology of weak convergence is itself a complete, separable, metric space [4. The Prohorov metric, for example, can be used to metrize this space. This metric has found many applications in information theory and stochastic control. Furthermore, there are well-known conditions to identify whether a family of probability measures is weakly compact 4]. For these reasons, one would like to work with weak convergence. However, as we will observe, weak convergence is insufficient in a general setup for obtaining continuity.

Before proceeding further, however, we look for conditions under which an optimal control policy exists; i.e, when the infimum in $\inf _{\gamma} E_{P}^{Q, \gamma}[c(X, U)]$ is a minimum. The following simple result is proved in the Appendix.

TheOREm 3.1. Suppose assumptions A3 and A4 hold. Then, there exists an optimal control policy for any channel $Q$.

REMARK. The assumptions that $c$ is bounded and $\mathbb{U}$ is compact can be weakened in the preceding theorem. For example, one can prove the same result by assuming that $\mathbb{U}=\mathbb{R}^{k}, \lim _{\|u\| \rightarrow \infty} c(x, u)=\infty$ for all $x, c(x, u)$ is lower semi-continuous on $\mathbb{U}$ for every $x$, and there exists $u_{0}$ such that $\int c\left(x, u_{0}\right) P(d x)<\infty$.

\subsection{Weak convergence.}

3.1.1. Absence of continuity under weak convergence. The following counterexample demonstrates that $J(P, Q)$ may not be continuous under weak convergence of channels even for continuous cost functions and compact $\mathbb{X}, \mathbb{Y}$, and $\mathbb{U}$. Note that the absence of continuity here is also implied by a less elementary counterexample for setwise convergence in Section 3.2.1

Let $\mathbb{X}=\mathbb{Y}=\mathbb{U}=[a, b]$ for some $a, b \in \mathbb{R}, a<b$. Suppose the cost is given as $c(x, u)=(x-u)^{2}$ and assume that $P$ is a discrete distribution with two atoms:

$$
P=\frac{1}{2} \delta_{a}+\frac{1}{2} \delta_{b}
$$


where $\delta_{a}$ is the delta measure at point $a$, that is, $\delta_{a}(A)=1_{\{a \in A\}}$ for every Borel set $A$, where $1_{E}$ denotes the indicator function of event $E$. Let $\left\{Q_{n}\right\}$ be a sequence of channels given by

$$
Q_{n}(\cdot \mid x)=\left\{\begin{array}{lll}
\delta_{a+\frac{1}{n}} & \text { if } & x \geq a+\frac{1}{n} \\
\delta_{a} & \text { if } & x<a+\frac{1}{n}
\end{array}\right.
$$

In this case, the optimal control policy, which is unique up to changes in points of measure zero, is

$$
\gamma_{n}(y)=a 1_{\left\{y<a+\frac{1}{n}\right\}}+b 1_{\left\{y \geq a+\frac{1}{n}\right\}}, \quad n \in \mathbb{N}, \quad n \geq \frac{1}{b-a},
$$

leading to a cost of 0 . We observe that the limit of the sequence $\left\{Q_{n}(\cdot \mid x)\right\}$ is given by

$$
Q(\cdot \mid x)=\delta_{a} \quad \text { for all } x \in \mathbb{R} .
$$

Thus, by Lemma 2.2, $Q_{n} \rightarrow Q$ weakly at input $P$. However, the limit of the sequence of channels cannot distinguish between the inputs, since the channel output always equals $a$. Thus, even though

$$
J\left(P, Q_{n}\right)=0, \quad \text { for all } n \geq \frac{1}{b-a},
$$

the cost of $Q=\lim _{n} Q_{n}$ is

$$
J(P, Q)=\frac{(b-a)^{2}}{4}
$$

since, letting $(X, Y) \sim P Q$, we have $\gamma(y)=E[X \mid Y=y]=(b+a) / 2$ for all $y$.

\subsubsection{Upper semi-continuity under weak convergence.}

ThEOREM 3.2. Suppose assumptions A1 and A5 hold. If $\left\{Q_{n}\right\}$ is a sequence of channels converging weakly at input $P$ to a channel $Q$, then

$$
\limsup _{n \rightarrow \infty} J\left(P, Q_{n}\right) \leq J(P, Q),
$$

that is, $J(P, Q)$ is upper semi-continuous on $\mathcal{Q}$ under weak convergence.

Proof. Let $\mu$ be an arbitrary probability measure on $(\mathbb{X} \times \mathbb{Y}, \mathcal{B}(\mathbb{X} \times \mathbb{Y}))$ and let $\mu_{\mathbb{Y}}$ be its second marginal, i.e., $\mu_{\mathbb{Y}}(A)=\mu(\mathbb{X} \times A)$ for $A \in \mathcal{B}(\mathbb{Y})$. Let $g \in \mathcal{G}$ be arbitrary. By Lusin's theorem [27, Thm. 2.24] there is a continuous function $f: \mathbb{Y} \rightarrow \mathbb{U}$ such that

$$
\mu_{\mathbb{Y}}\{y: f(y) \neq g(y)\}<\epsilon .
$$

Letting $B=\{y: f(y) \neq g(y)\}$ we obtain

$$
\int|c(x, g(y))-c(x, f(y))| \mu(d x, d y)=\int_{\mathbb{X} \times B}|c(x, g(y))-c(x, f(y))| \mu(d x, d y)
$$

\footnotetext{
${ }^{1}$ Lusin's theorem as stated in 27 implies the statement for $\mathbb{U}=\mathbb{R}$. The extension to the case $\mathbb{U}=\mathbb{R}^{K}$ is straightforward. If $\mathbb{U}$ is any closed and convex subset of $\mathbb{R}^{K}$, then there is a continuous function $\pi: \mathbb{R}^{K} \rightarrow \mathbb{U}$ such that $\pi(u)=u$ on $\mathbb{U}$ (the metric projection onto $\mathbb{U}$ ). Then $\hat{f}=\pi \circ f$ is the desired continuous mapping from $\mathbb{Y}$ into $\mathbb{U}$.
} 


$$
<\epsilon \cdot c^{*}
$$

where $c^{*}=\sup _{x, u} c(x, u)<\infty$ by assumption A1, so that

$$
\int c(x, f(y)) \mu(d x, d y)<\int c(x, g(y)) \mu(d x, d y)+c^{*} \epsilon .
$$

Let $\mathcal{C}$ be the set of continuous functions from $\mathbb{Y}$ into $\mathbb{U}$, define

$$
j(\mu, \mathcal{C})=\inf _{\gamma \in \mathcal{C}} \int c(x, \gamma(y)) \mu(d x, d y), \quad j(\mu, \mathcal{G})=\inf _{\gamma \in \mathcal{G}} \int c(x, \gamma(y)) \mu(d x, d y)
$$

and note that $j(\mu, \mathcal{C}) \geq j(\mu, \mathcal{G})$ since $\mathcal{C} \subset \mathcal{G}$. By (3.3), $j(\mu, \mathcal{C})$ is upper bounded by the right-hand-side of (3.3). Since $g$ in (3.3) was arbitrary, we obtain $j(\mu, \mathcal{C}) \leq$ $j(\mu, \mathcal{G})+c^{*} \epsilon$, which in turn implies $j(\mu, \mathcal{C}) \leq j(\mu, \mathcal{G})$ since $\epsilon>0$ was arbitrary. Hence $j(\mu, \mathcal{C})=j(\mu, \mathcal{G})$.

Applying the above first to $P Q_{n}$ and then to $P Q$, we obtain

$$
\begin{aligned}
\limsup _{n \rightarrow \infty} \inf _{\gamma \in \mathcal{G}} \int c(x, \gamma(y)) P Q_{n}(d x, d y) & =\limsup _{n \rightarrow \infty} \inf _{f \in \mathcal{C}} \int c(x, f(y)) P Q_{n}(d x, d y) \\
& \leq \inf _{f \in \mathcal{C}} \limsup _{n \rightarrow \infty} \int c(x, f(y)) P Q_{n}(d x, d y) \\
& =\inf _{f \in \mathcal{C}} \int c(x, f(y)) P Q(d x, d y) \\
& =\inf _{\gamma \in \mathcal{G}} \int c(x, \gamma(y)) P Q(d x, d y)
\end{aligned}
$$

where the next to last equality holds since $P Q_{n}$ converges weakly to $P Q$.

\subsection{Continuity properties under setwise convergence.}

3.2.1. Absence of continuity under setwise convergence. The following counterexample demonstrates that $J(P, Q)$ may not be continuous under setwise convergence of channels even for continuous cost functions and compact $\mathbb{X}, \mathbb{Y}$, and $\mathbb{U}$.

Let $\mathbb{X}=\mathbb{Y}=\mathbb{U}=[0,1]$. Assume that $X$ has distribution

$$
P=\frac{1}{2} \delta_{0}+\frac{1}{2} \delta_{1}
$$

Let $Q(\cdot \mid x)=U([0,1])$ for all $x$, so that if $(X, Y) \sim P Q$, then $Y$ is independent of $X$ and has the uniform distribution on $[0,1]$. Let $c(x, u)=(x-u)^{2}$.

By independence, $E[X \mid Y]=E[X]=1 / 2$, so

$$
\begin{aligned}
J(P, Q) & =\min _{\gamma \in \mathcal{G}} E\left[(X-\gamma(Y))^{2}\right]=E\left[(X-E[X \mid Y])^{2}\right] \\
& =\frac{1}{2}\left(1-\frac{1}{2}\right)^{2}+\frac{1}{2}\left(0-\frac{1}{2}\right)^{2}=\frac{1}{4} .
\end{aligned}
$$

For $n \in \mathbb{N}$ and $k=1, \ldots, n$ consider the intervals

$$
L_{n k}=\left[\frac{2 k-2}{2 n}, \frac{2 k-1}{2 n}\right), \quad R_{n k}=\left[\frac{2 k-1}{2 n}, \frac{2 k}{2 n}\right)
$$


and define the "square wave" function

$$
h_{n}(t)=\sum_{k=1}^{n}\left(1_{\left\{t \in L_{n k}\right\}}-1_{\left\{t \in R_{n k}\right\}}\right) .
$$

Since $\int_{0}^{1} h_{n}(t) d t=0$ and $\left|h_{n}(t)\right| \leq 1$, the function

$$
f_{n}(t)=\left(1+h_{n}(t)\right) 1_{\{t \in[0,1]\}}
$$

is a probability density function. Furthermore, the proof of the Riemann-Lebesgue lemma (for example [31, Thm. 12.21) can be used almost verbatim to show that

$$
\lim _{n \rightarrow \infty} \int_{0}^{1} h_{n}(t) g(t) d t=0 \quad \text { for all } g \in L_{1}([0,1], \mathbb{R})
$$

and therefore

$$
\lim _{n \rightarrow \infty} \int_{0}^{1} f_{n}(t) g(t) d t=\int_{0}^{1} g(t) d t \quad \text { for all } g \in L_{1}([0,1], \mathbb{R}) .
$$

In particular, we obtain that the sequence of probability measures induced by the sequence $\left\{f_{n}\right\}$ converges setwise to $U([0,1])$.

Now, for every $n$, define a channel as

$$
Q_{n}(\cdot \mid x)= \begin{cases}U([0,1]), & x=0 \\ \sim f_{n}, & x=1\end{cases}
$$

Then $Q_{n}(\cdot \mid x) \rightarrow Q$ setwise for $x=0$ and $x=1$, and thus $P Q_{n} \rightarrow P U([0,1])$ setwise. However, letting $\left(X, Y_{n}\right) \sim P Q_{n}$, a simple calculation shows that the optimal policy for $P Q_{n}$ is

$$
\gamma_{n}(y)=E\left[X \mid Y_{n}=y\right]= \begin{cases}0, & y \in \bigcup_{k=1}^{n} R_{n k} \\ \frac{2}{3}, & y \in \bigcup_{k=1}^{n} L_{n k}\end{cases}
$$

and therefore for every $n \in \mathbb{N}$

$$
\begin{aligned}
J\left(P, Q_{n}\right) & =\min _{\gamma \in \mathcal{G}} E\left[\left(X-\gamma\left(Y_{n}\right)\right)^{2}\right] \\
& =\frac{1}{2} \int_{0}^{1}\left(0-\gamma_{n}(y)\right)^{2} d y+\frac{1}{2} \int_{0}^{1}\left(1-\gamma_{n}(y)\right)^{2} f_{n}(y) d y \\
& =\frac{1}{6} .
\end{aligned}
$$

Thus, the optimal cost value is not continuous under setwise convergence.

\subsubsection{Upper semi-continuity under setwise convergence.}

THEOREM 3.3. Under assumption A2 the optimal cost

$$
J(P, Q):=\inf _{\gamma} E_{P}^{Q, \gamma}[c(X, U)]
$$

is sequentially upper semi-continuous on the set of communication channels $\mathcal{Q}$ under setwise convergence. 
Proof. Let $\left\{Q_{n}\right\}$ converge setwise to $Q$ at input $P$. Then

$$
\begin{aligned}
\limsup _{n \rightarrow \infty} \inf _{\gamma \in \mathcal{G}} \int c(x, \gamma(y)) P Q_{n}(d x, d y) & \leq \inf _{\gamma \in \mathcal{G}} \limsup _{n \rightarrow \infty} \int c(x, \gamma(y)) P Q_{n}(d x, d y) \\
& =\inf _{\gamma \in \mathcal{G}} \int c(x, \gamma(y)) P Q(d x, d y),
\end{aligned}
$$

where the equality holds since $c$ is bounded.

\subsection{Continuity under total variation.}

THEOREM 3.4. Under assumption A2 the optimal cost $J(P, Q)$ is is continuous on the set of communication channels $\mathcal{Q}$ under under the topology of total variation.

Proof. Assume $Q_{n} \rightarrow Q$ in total variation at input $P$. Let $\epsilon>0$ and pick the $\epsilon$-optimal policies $\gamma_{n}$ and $\gamma$ under channels $Q_{n}$ and $Q$, respectively. That is, letting $\hat{J}\left(Q^{\prime}, \gamma^{\prime}\right)=E_{P}^{Q^{\prime}, \gamma^{\prime}}[c(X, U)]$ for any $\gamma^{\prime} \in \mathcal{G}$ and $Q^{\prime} \in \mathcal{Q}$, we have $\hat{J}\left(Q_{n}, \gamma_{n}\right)<$ $J\left(P, Q_{n}\right)+\epsilon$ and $\hat{J}(Q, \gamma)<J(P, Q)+\epsilon$.

Considering first the case $J\left(P, Q_{n}\right)<J(P, Q)$, we have

$$
\begin{aligned}
J(P, Q)-J\left(P, Q_{n}\right) & \leq J(P, Q)-\hat{J}\left(Q_{n}, \gamma_{n}\right)+\epsilon \\
& \leq \hat{J}\left(Q, \gamma_{n}\right)-\hat{J}\left(Q_{n}, \gamma_{n}\right)+\epsilon .
\end{aligned}
$$

By a symmetric argument it follows that

$$
\left|J(P, Q)-J\left(P, Q_{n}\right)\right| \leq \max \left(\hat{J}\left(Q, \gamma_{n}\right)-\hat{J}\left(Q_{n}, \gamma_{n}\right), \hat{J}\left(Q_{n}, \gamma\right)-\hat{J}(Q, \gamma)\right)+\epsilon
$$

Now, since $c$ is bounded, it follows from (2.1) that for any $\gamma^{\prime} \in \mathcal{G}$,

$$
\begin{aligned}
\left|\hat{J}\left(Q_{n}, \gamma^{\prime}\right)-\hat{J}\left(Q, \gamma^{\prime}\right)\right| & =\left|\int c\left(x, \gamma^{\prime}(y)\right) P Q_{n}(d x, d y)-\int c\left(x, \gamma^{\prime}(y)\right) P Q(d x, d y)\right| \\
& \leq\|c\|_{\infty}\left\|P Q_{n}-P Q\right\|_{T V} .
\end{aligned}
$$

This and (3.6) imply $\left|J\left(P, Q_{n}\right)-J(P, Q)\right| \leq\|c\|_{\infty}\left\|P Q_{n}-P Q\right\|_{T V}+\epsilon$. Since $\epsilon>0$ was arbitrary, we obtain $\left|J\left(P, Q_{n}\right)-J(P, Q)\right| \leq\|c\|_{\infty}\left\|P Q_{n}-P Q\right\|_{T V}$. Since $\| P Q_{n}-$ $P Q \|_{T V} \rightarrow 0$ by Lemma 2.2, we obtain $J\left(P, Q_{n}\right) \rightarrow J(P, Q)$ as claimed.

4. Problem P2: Existence of optimal channels. Here we study characterizations of compactness which will be useful in obtaining existence results.

The discussion on weak convergence showed us that weak convergence does not induce a strong enough topology, i.e., under which useful continuity properties can be obtained. In the following, we will obtain conditions for compactness for the other two convergence notions, that is, for setwise convergence and total variation. We note that in the topologies induced by these three modes of convergence, notions of compactness and sequential compactness coincide (for total variation and weak convergence this follows from metrizability; for setwise convergence see [6, Thm. 4.7.25]).

We first discuss setwise convergence. A set of probability measures $\mathcal{M}$ on some measurable space is said to be setwise precompact if every sequence in $\mathcal{M}$ has a subsequence converging setwise to a probability measure (not necessarily in $\mathcal{M}$ ). For two finite measures $\nu$ and $\mu$ defined on the same measurable space we write $\nu \leq \mu$ if $\nu(A) \leq \mu(A)$ for all measurable $A$.

We have the the following condition for setwise (pre)compactness:

Lemma 4.1 (6, Thm. 4.7.25]). Let $\mu$ be a finite measure on a measurable space $(\mathbb{T}, \mathcal{A})$. Assume a set of probability measures $\Psi \subset \mathcal{P}(\mathbb{T})$ satisfies

$$
P \leq \mu, \quad \text { for all } P \in \Psi .
$$


Then $\Psi$ is setwise precompact.

As before, $P Q \in \mathcal{P}(\mathbb{X} \times \mathbb{Y})$ denotes the joint probability measure induced by input $P$ and channel $Q$, where $\mathbb{X}=\mathbb{R}^{n}$ and $\mathbb{Y}=\mathbb{R}^{m}$. A simple consequence of the preceding majorization criterion is the following.

LEMMA 4.2. Let $\nu$ be a finite measure on $\mathcal{B}(\mathbb{X} \times \mathbb{Y})$ and let $P$ be a probability measure on $\mathcal{B}(\mathbb{X})$. Suppose $\mathcal{Q}$ is a set of channels such that

$$
P Q \leq \nu, \quad \text { for all } Q \in \mathcal{Q} .
$$

Then $\mathcal{Q}$ is setwise precompact at input $P$ in the sense that any sequence in $\mathcal{Q}$ has a subsequence $\left\{Q_{n}\right\}$ such that $Q_{n} \rightarrow Q$ setwise at input $P$ for some channel $Q$.

Proof. By Lemma 4.1, the set of joint measures $\mathcal{M}=\{P Q: Q \in \mathcal{Q}\}$ is setwise precompact, that is, any sequence in $\mathcal{M}$ has a subsequence $\left\{P Q_{n}\right\}$ converging to some $\hat{P}$ setwise. Furthermore, since the first marginal of $P Q$ is $P$ for all $n$, the first marginal of $\hat{P}$ is also $P$ (since $P Q_{n}(A \times \mathbb{X}) \rightarrow \hat{P}(A \times \mathbb{X})$ for all $\left.A \in \mathcal{B}(\mathbb{X})\right)$. Now let $Q$ be a regular conditional probability measure satisfying $\hat{P}=P Q$.

For a probability density function $p$ on $\mathbb{R}^{N}$ we let $P_{p}$ denote the induced probability measure: $P_{p}(A)=\int_{A} p(x) d x, A \in \mathcal{B}\left(\mathbb{R}^{N}\right)$. The next lemma gives a sufficient condition for precompactness under total variation.

Lemma 4.3. Let $\mu$ be a finite Borel measure on $\mathbb{R}^{N}$ and let $\mathcal{F}$ be an equicontinuous and uniformly bounded family of probability density functions. Define $\Psi \subset \mathcal{P}\left(\mathbb{R}^{N}\right)$ by

$$
\Psi=\left\{P_{p}: P_{p} \leq \mu, p \in \mathcal{F}\right\} .
$$

Then $\Psi$ is precompact under total variation.

Proof. By Lemma 4.1, $\Psi$ is setwise precompact and thus any sequence in $\Psi$ has a subsequence $\left\{P_{n}\right\}$ such that $P_{n} \rightarrow P$ setwise for some $P \in \mathcal{P}\left(\mathbb{R}^{N}\right) . P$ is clearly absolutely continuous with respect to the Lebesgue measure on $\mathbb{R}^{N}$, and so it admits a density $p$.

Let $p_{n}$ be the density of $P_{n}$. It suffices to show that

$$
\lim _{n \rightarrow \infty}\left\|p_{n}-p\right\|_{1}=0
$$

since $\left\|p_{n}-p\right\|_{T V}=2\left\|p_{n}-p\right\|_{1}=2 \int\left|p_{n}(x)-p(x)\right| d x$.

Pick a sequence of compact sets $K_{j} \subset \mathbb{R}^{N}$ such that $K_{j} \subset K_{j+1}$ for all $j \in \mathbb{N}$, and $\bigcup_{j} K_{j}=\mathbb{R}^{N}$. Since the collection of densities $\left\{p_{n}\right\}$ is uniformly bounded and equicontinuous, it is precompact in the supremum norm on each $K_{j}$ by the ArzelàAscoli theorem [13]. Thus there exist subsequences $\left\{p_{n_{k}^{j}}\right\}$ such that

$$
\lim _{k \rightarrow \infty} \sup _{x \in K_{j}}\left|p_{n_{k}^{j}}(x)-p^{j}(x)\right|=0
$$

for some continuous $p^{j}: K_{j} \rightarrow[0, \infty)$.

Since the $K_{j}$ are nested, one can choose $\left\{p_{n_{k}^{j+1}}\right\}$ to be a subsequence of $\left\{p_{n_{k}^{j}}\right\}$ for all $j \in \mathbb{N}$. Then $p^{j+1}$ coincides with $p^{j}$ on $K_{j}$ and we can define $\hat{p}$ on $\mathbb{R}^{N}$ by setting $\hat{p}(x)=p^{j}(x), x \in K_{j}$. We can now use Cantor's diagonal method to pick an increasing sequence of integers $\left\{m_{i}\right\}$ which is a subsequence of each $\left\{n_{k}^{j}\right\}$, and thus

$$
\lim _{i \rightarrow \infty} p_{m_{i}}(x)=\hat{p}(x), \quad \text { for all } x \in \mathbb{R}^{N} .
$$

Note that by construction the convergence is uniform on each $K_{j}$ (and $\hat{p}$ is continuous). By uniform convergence $P_{p_{m_{i}}}(A) \rightarrow P_{\hat{p}}(A)$ for all Borel subsets $A$ of $K_{j}$. The setwise 
convergence of $P_{n}$ to $P_{p}$ implies $P_{p_{m_{i}}}(A) \rightarrow P_{p}(A)$ for all Borel sets, so we must have $p=\hat{p}$ almost everywhere. This and (4.2) imply via Scheffé's theorem [5] that

$$
\left\|p_{m_{j}}-p\right\|_{1} \rightarrow 0
$$

which completes the proof.

The next result is an analogue of Lemma 4.2 and has an essentially identical proof.

LEMMA 4.4. Let $\mathcal{Q}$ be a set of channels such that $\{P Q: Q \in \mathcal{Q}\}$ is a precompact set of probability measures under total variation. Then $\mathcal{Q}$ is precompact under total variation at input $P$.

The following theorem, when combined with the preceding results, gives sufficient conditions for the existence of best and worst channels when the given family of channels $\mathcal{Q}$ is closed under the appropriate convergence notion.

THEOREM 4.5. Recall problem P2.

(i) There exist a worst channel in $\mathcal{Q}$, that is, a solution for the maximization problem

$$
\sup _{Q \in \mathcal{Q}} J(P, Q)=\sup _{Q \in \mathcal{Q}} \inf _{\gamma} E_{P}^{Q, \gamma} E[c(X, U)]
$$

when the set $\mathcal{Q}$ is weakly compact and assumptions $\mathrm{A} 1, \mathrm{~A} 4$, and $\mathrm{A} 5$ hold.

(ii) There exist a worst channel in $\mathcal{Q}$ when the set $\mathcal{Q}$ is setwise compact and assumption A2 holds.

(iii) There exist best and worst channels in $\mathcal{Q}$, that is, solutions for the minimization problem $\inf _{Q \in \mathcal{Q}} J(P, Q)$ and the maximization problem $\sup _{Q \in \mathcal{Q}} J(P, Q)$ when the set $\mathcal{Q}$ is compact under total variation and assumption $\mathrm{A} 2$ holds.

Proof. Under the stated conditions, we have upper semi-continuity or continuity (Theorems 3.2, 3.3 and 3.4) under the corresponding topologies. By compactness, the existence of the cost maximizing (worst) channel follows when $J(P, Q)$ is uppersemicontinuous, while the existence of the cost minimizing (best) channel follows when $J(P, Q)$ is continuous in $Q$. $\square$

REMARK. The existence of worst channels is useful for the robust control or gametheoretic approach to optimization problems. If the problem is formulated as a game where the uncertainty in the set is regarded as a maximizer and the controller is the minimizer, one could search for a max-min solution, which we prove to exist. One could also look for min-max solutions, a topic which we leave as a future research topic. We note that, in information theory, problems of similar nature have been considered in the context of mutual information games 9 .

5. Application: quantizers as a class of channels. Here we consider the problem of convergence and optimization of quantizers. We start with the definition of a quantizer.

Definition 5.1. An $M$-cell vector quantizer, $q$, is a (Borel) measurable mapping from $\mathbb{X}=\mathbb{R}^{n}$ to the finite set $\{1,2, \ldots, M\}$, characterized by a measurable partition $\left\{B_{1}, B_{2}, \ldots, B_{M}\right\}$ such that $B_{i}=\{x: q(x)=i\}$ for $i=1, \ldots, M$. The $B_{i}$ are called the cells (or bins) of $q$.

REMARKS.

(i) For later convenience we allow for the possibility that some of the cells of the quantizer are empty.

(ii) Traditionally, in source coding theory, a quantizer is a mapping $q: \mathbb{R}^{n} \rightarrow \mathbb{R}$ with a finite range. Thus $q$ is defined by a partition and a reconstruction value in $\mathbb{R}^{n}$ 
for each cell in the partition. That is, for given cells $\left\{B_{1}, \ldots, B_{M}\right\}$ and reconstruction values $\left\{c_{1}, \ldots, c_{M}\right\} \subset \mathbb{R}^{n}$, we have $q(x)=c_{i}$ if and only if $x \in B_{i}$. In our definition, we do not include the reconstruction values.

A quantizer $q$ with cells $\left\{B_{1}, \ldots, B_{M}\right\}$, however, can also be characterized as a stochastic kernel $Q$ from $\mathbb{X}$ to $\{1, \ldots, M\}$ ) defined by

$$
Q(i \mid x)=1_{\left\{x \in B_{i}\right\}}, \quad i=1, \ldots, M
$$

so that $q(x)=\sum_{i=1}^{M} Q(i \mid x)$. We denote by $\mathcal{Q}_{D}(M)$ the space of all $M$-cell quantizers represented in the channel form. In addition, we let $\mathcal{Q}(M)$ denote the set of (Borel) stochastic kernels from $\mathbb{X}$ to $\{1, \ldots, M\}$, i.e., $Q \in \mathcal{Q}(M)$ if and only if $Q(\cdot \mid x)$ is probability distribution on $\{1, \ldots, M\}$ for all $x \in \mathbb{X}$, and $Q(i \mid \cdot)$ is Borel measurable for all $i=1, \ldots, M$. Note that $\mathcal{Q}_{D}(M) \subset \mathcal{Q}(M)$, and by our definition $\mathcal{Q}_{D}(M-1) \subset$ $\mathcal{Q}_{D}(M)$ for all $M \geq 2$. We note that elements of $\mathcal{Q}(M)$ are sometimes referred to in the literature as random quantizers.

LEMma 5.2. The set of quantizers $\mathcal{Q}_{D}(M)$ is setwise precompact at any input $P$.

Proof. Proof follows from Lemma 4.2 and the interpretation above regarding a quantizer as a channel. In particular, a majorizing finite measure $\nu$ is obtained by defining $\nu=P \times \lambda$, where $\lambda$ is the counting measure on $\{1, \ldots, M\}$ (note that $\left.\nu\left(\mathbb{R}^{n} \times\{1, \ldots, M\}\right)=M\right)$. Then for any measurable $B \subset \mathbb{R}^{n}$ and $i=1, \ldots, M$, we have $\nu(B \times\{i\})=P(B) \lambda(\{i\})=P(B)$ and so

$$
P Q(B \times\{i\})=P\left(B \cap B_{i}\right) \leq P(B)=\nu(B \times\{i\}) .
$$

Since any measurable $D \subset \mathbb{X} \times\{1, \ldots, M\}$ can be written as the disjoint union of the sets $D_{i} \times\{i\}, i=1, \ldots, M$, with $D_{i}=\{x \in \mathcal{X}:(x, i) \in D\}$, the above implies $P Q(D) \leq \nu(D)$.

The following simple lemma provides a useful formula.

Lemma 5.3. A sequence $\left\{Q_{n}\right\}$ in $\mathcal{Q}(M)$ converges to a $Q$ in $\mathcal{Q}(M)$ setwise at input $P$ if and only if

$$
\int_{A} Q_{n}(i \mid x) P(d x) \rightarrow \int_{A} Q(i \mid x) P(d x) \quad \text { for all } A \in \mathcal{B}(\mathbb{X}) \text { and } i=1, \ldots, M .
$$

Proof. The lemma follows by noticing that for any $Q \in \mathcal{Q}(M)$ and measurable $D \subset \mathbb{X} \times\{1, \ldots, M\}$,

$$
P Q(D)=\int_{D} Q(d y \mid x) P(d x)=\sum_{i=1}^{M} \int_{D_{i}} Q(i \mid x) P(d x)
$$

where $D_{i}=\{x \in \mathcal{X}:(x, i) \in D\}$.

The following counterexample shows that the space of quantizers $\mathcal{Q}_{D}(M)$ is not closed under setwise convergence:

Let $\mathbb{X}=[0,1]$ and $P$ the uniform distribution on $[0,1]$. Recall the definition $L_{n k}=\left[\frac{2 k-2}{2 n}, \frac{2 k-1}{2 n}\right)$ in (3.4) and let $B_{n, 1}=\bigcup_{i=1}^{n} L_{n k}$ and $B_{n, 2}=[0,1] \backslash B_{n, 1}$. Define $\left\{Q_{n}\right\}$ as the sequence of 2-cell quantizers given by

$$
Q_{n}(1 \mid x)=1_{\left\{x \in B_{n, 1}\right\}}, \quad Q_{n}(2 \mid x)=1_{\left\{x \in B_{n, 2}\right\}} .
$$


Then (3.5) implies that for all $A \in \mathcal{B}([0,1])$,

$$
\lim _{n \rightarrow \infty} \int_{A} Q_{n}(d y \mid x) P(d x)=\lim _{n \rightarrow \infty} \int_{0}^{1} \frac{1}{2} f_{n}(t) d t=\frac{1}{2} P(A),
$$

and thus, by Lemma 5.3, $Q_{n}$ converges setwise to $Q$ given by $Q(1 \mid x)=Q(2 \mid x)=\frac{1}{2}$ for all $x \in[0,1]$. However, $Q$ is not a (deterministic) quantizer.

DEFINITION 5.4. The class of finitely randomized quantizers $\mathcal{Q}_{F R}(M)$ is the convex hull of $\mathcal{Q}_{D}(M)$, i.e., $Q \in \mathcal{Q}_{F R}(M)$ if and only if there exist $k \in \mathbb{N}, Q_{1}, \ldots, Q_{k} \in$ $\mathcal{Q}_{D}(M)$, and $\alpha_{1}, \ldots, \alpha_{k} \in[0,1]$ with $\sum_{i=1}^{k} \alpha_{i}=1$, such that

$$
Q(i \mid x)=\sum_{j=1}^{k} \alpha_{j} Q_{j}(i \mid x), \quad \text { for all } i=1, \ldots, M \text { and } x \in \mathbb{X} .
$$

The next result shows that $\mathcal{Q}_{R}(M)$ is the closure of the convex hull of $\mathcal{Q}_{D}(M)$.

TheOREM 5.5. For any $Q \in \mathcal{Q}(M)$ there exists a sequence $\left\{\hat{Q}_{n}\right\}$ of finitely randomized quantizers in $\mathcal{Q}_{F R}(M)$ which converges to $Q$ setwise at any input $P$.

Proof. We will prove the existence of a sequence $\left\{\hat{Q}_{n}\right\}$ in $\mathcal{Q}_{F R}(M)$ such that $\hat{Q}_{n}(\cdot \mid x) \rightarrow Q(\cdot \mid x)$ setwise for all $x \in \mathbb{X}$.

Let $\mathcal{P}_{M}=\left\{z \in \mathbb{R}^{M}: z_{1}+\cdots+z_{M}=1, z_{i} \geq 0, i=1, \ldots, M\right\}$ denote the probability simplex in $\mathbb{R}^{M}$ and note that each $Q \in \mathcal{Q}(\mathcal{M})$ is uniquely represented by the function $Q^{v}: \mathbb{X} \rightarrow \mathcal{P}_{M}$ defined by

$$
Q^{v}(x)=(Q(1 \mid x), Q(2 \mid x), \ldots, Q(M \mid x)) .
$$

For a positive integer $n$ let $\mathcal{P}_{M, n}$ be the collection of probability vectors in $\mathcal{P}_{M}$ with rational components having common denominator $n$, i.e.,

$$
\mathcal{P}_{M, n}=\left\{z \in \mathcal{P}_{M}: z_{i} \in\{0,1 / n, \ldots,(n-1) / n, 1\}, i=1, \ldots, M\right\} .
$$

Clearly, any $z \in \mathcal{P}_{M}$ can be approximated within error $1 / n$ in the $l_{\infty}$ sense by a member of $\mathcal{P}_{M, n}$, i.e.,

$$
\max _{z \in \mathcal{P}_{M}} \min _{z^{\prime} \in \mathcal{P}_{M, n}}\left\|z-z^{\prime}\right\|_{\infty}=\max _{z \in \mathcal{P}_{M}} \min _{z^{\prime} \in \mathcal{P}_{M, n}} \max _{i=1, \ldots, M}\left|z_{i}-z_{i}^{\prime}\right| \leq \frac{1}{n} .
$$

Breaking ties in a predetermined manner, we can make the selection of $z^{\prime}$ for a given $z$ unique, and thus define a Borel measurable mapping $q_{n}: \mathcal{P}_{M} \rightarrow \mathcal{P}_{M, n}$ such that $z^{\prime}=q_{n}(z)$ approximates $z$ in the above sense. Given $Q \in \mathcal{Q}(\mathcal{M})$, use this mapping to define $Q_{n} \in \mathcal{Q}(\mathcal{M})$ through the relation

$$
Q_{n}^{v}(x)=q_{n}\left(Q^{v}(x)\right) .
$$

(The measurability of $Q(i \mid x)$ in $x$ follows from the measurability of the mapping $q_{n}$.) Let $\left\{z^{(1)}, \ldots, z^{(L(n))}\right\}$ be an enumeration of those elements of $\mathcal{P}_{M, n}$ for which the sets

$$
S_{j}=\left\{x: Q_{n}^{v}(x)=z^{(j)}\right\}, \quad j=1, \ldots, L(n)
$$

are not empty (clearly, $\left.L(n) \leq(n+1)^{M}\right)$. Note that the $S_{i}$ form a Borel-measurable partition of $\mathbb{X}$ and we have

$$
u:=\left(z^{(1)}, z^{(2)}, \ldots, z^{L(n)}\right) \in\left(\mathcal{P}_{M}\right)^{L(n)}
$$


and

$$
Q_{n}^{v}(x)=z^{(j)} \quad \text { if } x \in S_{j} .
$$

Viewed as a subset of $\mathbb{R}^{M \cdot L(n)}$, the set $\left(\mathcal{P}_{M}\right)^{L(n)}$ is compact and convex and therefore by the Krein-Milman theorem (see, e.g., [3]) it is the closure of the convex hull of its extreme points. The set of extreme points of $\left(\mathcal{P}_{M}\right)^{L(n)}$ is $\left(\mathcal{E}_{M}\right)^{L(n)}$, where $\mathcal{E}_{M}=$ $\left\{e_{1}, \ldots, e_{M}\right\}$ is the standard basis for $\mathbb{R}^{M}$. In particular, we can find $u_{1}, \ldots, u_{N} \in$ $\left(\mathcal{E}_{M}\right)^{L(n)}$ and $\left(\alpha_{1}, \ldots, \alpha_{N}\right) \in \mathcal{P}_{N}$ such that $\left\|u-\sum_{k=1}^{N} \alpha_{k} u_{k}\right\| \leq \frac{1}{n}(\|\cdot\|$ denotes the standard Euclidean norm in any dimension). Since $u_{k}=\left(u_{k, 1}, \ldots, u_{k, L(n)}\right)$, where $u_{k, j} \in \mathcal{E}_{M}$ for all $k$ and $j$, we can define the deterministic quantizers $Q_{n, k} \in \mathcal{Q}_{D}(\mathcal{M})$, $k=1, \ldots, N$, by setting

$$
Q_{n, k}^{v}(x)=u_{k, j} \quad \text { if } x \in S_{j} .
$$

Putting things together, we obtain that

$$
\left\|Q_{n}^{v}(x)-\sum_{k=1}^{N} \alpha_{k} Q_{n, k}^{v}(x)\right\| \leq \frac{1}{n} \quad \text { for all } x \in \mathbb{X} .
$$

Define $\hat{Q}_{n} \in \mathcal{Q}(M)$ by

$$
\hat{Q}_{n}(i \mid x)=\sum_{k=1}^{N} \alpha_{k} Q_{n, k}(i \mid x)
$$

Combining (5.1) with $\left\|Q^{v}(x)-Q_{n}^{v}(x)\right\|_{\infty} \leq \frac{1}{n}$, we obtain

$$
\left|Q(i \mid x)-\hat{Q}_{n}(i \mid x)\right| \leq \frac{2}{n} \quad \text { for all } x \in \mathbb{X} \text { and } i=1, \ldots, M
$$

which implies that $\hat{Q}_{n}(\cdot \mid x) \rightarrow Q(\cdot \mid x)$ setwise for all $x \in \mathbb{X}$. Since each $\hat{Q}_{n}$ is a convex combination of deterministic quantizers in $\mathcal{Q}_{D}(M)$, the proof is complete.

The preceding theorem has important consequences in that it tells us that the space of deterministic quantizers is a "basis" for the space of communication channels between $\mathbb{X}$ and $\{1, \ldots, M\}$ in an appropriate sense. In the following we show that an optimal channel can be replaced with an optimal quantizer without any loss in performance.

Proposition 5.6. For any $Q \in \mathcal{Q}(M)$ there is a $Q^{\prime} \in \mathcal{Q}_{D}(M)$ with $J\left(P, Q^{\prime}\right) \leq$ $J(P, Q)$. If there exists an optimal channel in $\mathcal{Q}(M)$ for problem $\mathrm{P} 2$, then there is a quantizer in $\mathcal{Q}_{D}(M)$ that is optimal.

Proof. Only the first statement needs to be proved. We follow an argument common in the source coding literature (see, e.g., the Appendix of [33]).

For a policy $\gamma:\{1, \ldots, M\} \rightarrow \mathbb{U}=\mathbb{X}$ (with finite cost) define for all $i$,

$$
B_{i}=\{x: c(x, \gamma(i)) \leq c(x, \gamma(j)), \quad j=1, \ldots, M\} .
$$

Letting $B_{1}=\bar{B}_{1}$ and $B_{i}=\bar{B}_{i} \backslash \bigcup_{j=1}^{i-1} B_{j}, i=2, \ldots, M$, we obtain a partition $\left\{B_{i}, \ldots, B_{M}\right\}$ and a corresponding quantizer $Q^{\prime} \in \mathcal{Q}_{D}(M)$. It is easy to see that $E_{P}^{Q^{\prime}, \gamma}[c(X, U)] \leq E_{P}^{Q, \gamma}[c(X, U)]$ for any $Q \in \mathcal{Q}(M)$. 
The following shows that setwise convergence of quantizers implies convergence under total variation.

THEOREM 5.7. Let $\left\{Q_{n}\right\}$ be a sequence of quantizers in $\mathcal{Q}_{D}(M)$ which converges to a quantizer $Q \in \mathcal{Q}_{D}(M)$ setwise at $P$. Then, the convergence is also under total variation at $P$.

Proof. Let $B_{1}^{n}, \ldots, B_{M}^{n}$ be the cells of $Q_{n}$. Since $Q_{n} \rightarrow Q$ setwise at input $P$, we have $P Q_{n}(B \times\{i\}) \rightarrow P Q(B \times\{i\})$ for any $B \in \mathcal{B}(\mathbb{X})$. Since $P Q_{n}(B \times\{i\})=$ $\int_{B} 1_{\left\{x \in B_{i}^{n}\right\}} P(d x)$, we obtain

$$
P\left(B \cap B_{i}^{n}\right) \rightarrow P\left(B \cap B_{i}\right), \quad \text { for all } i=1, \ldots, M .
$$

If $B_{1}, \ldots, B_{M}$ are the cells of $Q$, the above implies $P\left(B_{j} \cap B_{i}^{n}\right) \rightarrow P\left(B_{j} \cap B_{i}\right)$ for all $i, j \in\{1, \ldots, M\}$. Since both $\left\{B_{i}^{n}\right\}$ and $\left\{B_{n}\right\}$ are partitions of $\mathbb{X}$, we obtain

$$
P\left(B_{i}^{n} \triangle B_{i}\right) \rightarrow 0 \text { for all } i=1, \ldots, M,
$$

where $B_{i}^{n} \triangle B=\left(B_{i}^{n} \backslash B\right) \cup\left(B \backslash B_{i}^{n}\right)$. Then we have

$$
\begin{aligned}
& \left\|P Q_{n}-P Q\right\|_{T V} \\
& =\sup _{f:\|f\|_{\infty} \leq 1}\left|\sum_{i=1}^{M}\left(\int_{\mathbb{X}} f(x, i) Q_{n}(i \mid x) P(d x)-\int_{\mathbb{X}} f(x, i) Q(i \mid x) P(d x)\right)\right| \\
& =\sup _{f:\|f\|_{\infty} \leq 1}\left|\sum_{i=1}^{M} \int_{\mathbb{X}} f(x, i)\left(1_{\left\{x \in B_{i}^{n}\right\}}-1_{\left\{x \in B_{i}^{n}\right\}}\right) P(d x)\right| \\
& \leq \sup _{f:\|f\|_{\infty} \leq 1} \sum_{i=1}^{M} \int_{B_{i}^{n} \triangle B_{i}}|f(x, i)| P(d x) \\
& \leq \sum_{i=1}^{M} P\left(B_{i}^{n} \triangle B_{i}\right) \rightarrow 0
\end{aligned}
$$

and convergence in total variation follows.

We next consider quantizers with convex codecells and an input distribution that is absolutely continuous with respect to the Lebesgue measure on $\mathbb{R}^{n}$ 18. Assume $Q \in \mathcal{Q}_{D}(M)$ with cells $B_{1}, \ldots, B_{M}$, each of which is a convex subset of $\mathbb{R}^{n}$. By the separating hyperplane theorem, there exist pairs of complementary closed half spaces $\left\{\left(H_{i, j}, H_{j, i}\right): 1 \leq i, j \leq M, i \neq j\right\}$ such that for all $i=1, \ldots, M$,

$$
B_{i} \subset \bigcap_{j \neq i} H_{i, j} .
$$

Each $\bar{B}_{i}:=\bigcap_{j \neq i} H_{i, j}$ is a closed convex polytope and by the absolute continuity of $P$ one has $P\left(\bar{B}_{i} \backslash B_{i}\right)=0$ for all $i=1, \ldots, M$. One can thus obtain a $(P-$ a.s $)$ representation of $Q$ by the $M(M-1) / 2$ hyperplanes $h_{i, j}=H_{i, j} \cap H_{j, i}$.

Let $\mathcal{Q}_{C}(M)$ denote the collection of $M$-cell quantizers with convex cells and consider a sequence $\left\{Q_{n}\right\}$ in $\mathcal{Q}_{C}(M)$. It can be shown (see the proof of Thm. 1 in [18]) that using an appropriate parametrization of the separating hyperplanes, a subsequence $Q_{n_{k}}$ can be can be chosen which converges to a $Q \in \mathcal{Q}_{C}(M)$ in the sense that $P\left(B_{i}^{n_{k}} \triangle B_{i}\right) \rightarrow 0$ for all $i=1, \ldots, M$, where the $B_{i}^{n_{k}}$ and the $B_{i}$ are the cells of $Q_{n_{k}}$ and $Q$, respectively. In view of (5.2), we obtain the following.

THEOREM 5.8. The set $\mathcal{Q}_{C}(M)$ is compact under total variation at any input measure $P$ that is absolutely continuous with respect to the Lebesgue measure on $\mathbb{R}^{n}$. 
We can now state an existence result for optimal quantization (problem P1).

TheOREM 5.9. Let $P$ be absolutely continuous and suppose the goal is to find the best quantizer $Q$ with $M$ cells minimizing $J(P, Q)=\inf _{\gamma} E_{P}^{Q, \gamma}(X, U)$ under assumption $\mathrm{A} 2$, where $Q$ is restricted to $\mathcal{Q}_{C}(M)$. Then an optimal quantizer exists.

Proof. Existence follows from Theorems 4.5 and 5.8, प

In the quantization literature finding an optimal quantizer means finding optimal codecells and corresponding reconstruction points. Our formulation does not require the existence of optimal reconstruction points (i.e., optimal policy $\gamma$ ). For cost functions of the form $c(x, u)=\|x-u\|^{p}$ for $x, u \in \mathbb{R}^{n}$ and some $p>0$, the cells of "good" quantizers will be convex by Lloyd-Max conditions of optimality; see 18 for further results on convexity of bins for entropy constrained quantization problems. We note that [1] also considered such cost functions for existence results on optimal quantizers; Graf and Luschgy [15] considered more general norm-based cost functions.

6. Multi-stage case. We consider the general case $T \in \mathbb{N}$. It should be observed that the effects of a control policy applied any given time-stage presents itself in two ways, in both the cost occurred at the given time-stage and the effect on the process distribution at future time-stages, which is known as the dual effect of control [2]

The next theorem shows the continuity of the optimal cost in the observation channel under some regularity conditions. Note that the existence of best and worst channels follows under an appropriate compactness condition as in Theorem 4.5 (iii). We need the following definition.

Definition 6.1. A sequence of channels $\left\{Q_{n}\right\}$ converges to a channel $Q$ uniformly in total variation if

$$
\lim _{n \rightarrow \infty} \sup _{x \in \mathbb{X}}\left\|Q_{n}(\cdot \mid x)-Q(\cdot \mid x)\right\|_{T V}=0 .
$$

Note that in the special but important case of additive observation channels, uniform convergence in total variation is equivalent to the weaker condition that $Q_{n}(\cdot \mid x) \rightarrow Q(\cdot \mid x)$ in total variation for each $x$. When the additive noise is absolutely continuous with respect to the Lebesgue measure, uniform convergence in total variation is equivalent to requiring that the noise density corresponding to $Q_{n}$ converges in the $L_{1}$ sense to the density corresponding to $Q$. For example, if the noise density is estimated from $n$ independent observations using any of the $L_{1}$ consistent density estimates described in e.g. [10, then the resulting $Q_{n}$ will converge (with probability one) uniformly in total variation.

TheOREm 6.2. Consider the cost function (1.2) with arbitrary $T \in \mathbb{N}$. Suppose assumption $\mathrm{A} 2$ holds. Then, the optimization problem $\mathrm{P} 1$ is continuous in the observation channel in the sense that if $\left\{Q_{n}\right\}$ is a sequence of channels converging to $Q$ uniformly in total variation, then

$$
\lim _{n \rightarrow \infty} J\left(P, Q_{n}\right)=J(P, Q) .
$$

Proof. Let $\epsilon>0$ and pick $\epsilon$-optimal policies $\Pi^{n}=\left\{\gamma_{0}^{n}, \gamma_{1}^{n}, \ldots, \gamma_{T-1}^{n}\right\}$ and $\Pi=$ $\left\{\gamma_{0}, \gamma_{1}, \ldots, \gamma_{T-1}\right\}$ for channels $Q_{n}$ and $Q$, respectively. That is, using the notation in (1.2), we have $J\left(P, Q_{n}, \Pi^{n}\right)<J\left(P, Q_{n}\right)+\epsilon$ and $J(P, Q, \Pi)<J(P, Q)+\epsilon$. The argument used to obtain (3.6) then gives

$$
\left|J(P, Q)-J\left(P, Q_{n}\right)\right|
$$




$$
\leq \max \left(J\left(P, Q, \Pi^{n}\right)-J\left(P, Q_{n}, \Pi^{n}\right), J\left(P, Q_{n}, \Pi\right)-J(P, Q, \Pi)\right)+\epsilon .
$$

We will show that both terms in the maximum converge to zero. First we consider the term

$$
J\left(P, Q^{n}, \Pi^{n}\right)-J\left(P, Q, \Pi^{n}\right)=\sum_{t=0}^{T-1} E_{P}^{Q^{n}, \Pi^{n}}\left[c\left(X_{t}, U_{t}\right)\right]-E_{P}^{Q, \Pi^{n}}\left[c\left(X_{t}, U_{t}\right)\right] .
$$

Under policy $\Pi^{n}=\left\{\gamma_{0}^{n}, \gamma_{1}^{n}, \ldots, \gamma_{T-1}^{n}\right\}$, we have $U_{t}=\gamma_{t}^{n}\left(Y_{[0, t]}, U_{[0, t-1]}\right)$. We absorb in the notation the dependence of $U_{t}$ on $\gamma_{0}^{n}, \ldots, \gamma_{t-1}^{n}$ and write $U_{t}=\gamma_{t}^{n}\left(Y_{[0, t]}\right)$.

For $t=0, \ldots, T-1$ and $k=0, \ldots, t$ define $\zeta_{k, t}^{n}: \mathbb{X}^{k} \times \mathbb{Y}^{k} \rightarrow \mathbb{R}$ by setting

$$
\zeta_{t, t}^{n}\left(x_{[0, t]}, y_{[0, t]}\right):=c\left(x_{t}, \gamma_{t}^{n}\left(y_{[0, t]}\right)\right.
$$

and defining recursively for $k=t-1, \ldots, 0$

$$
\zeta_{k, t}^{n}\left(x_{[0, k]}, y_{[0, k]}\right):=\int P\left(d x_{k+1} \mid x_{k}, \gamma_{k}^{n}\left(y_{[0, k]}\right)\right) Q_{n}\left(d y_{k+1} \mid x_{k+1}\right) \zeta_{k+1, t}^{n}\left(x_{[0, k+1]}, y_{[0, k+1]}\right) .
$$

Note that $\left\|\zeta_{t, t}^{n}\right\|_{\infty} \leq\|c\|_{\infty}$ and thus $\left\|\zeta_{k, t}^{n}\right\|_{\infty} \leq\|c\|_{\infty}$ for all $k=t-1, \ldots, 0$.

Fix $0 \leq k \leq t$ and consider a system such that the observation channel is $Q$ at stages $0, \ldots, k-1$ and $Q_{n}$ at stages $k, k+1, \ldots, t$. Let $\mu_{k}^{n}$ denote the distribution of the resulting process segment $\left(X_{[0, k]}, Y_{[0, k]}\right)$ under policy $\Pi^{n}$ (by definition $\left.\mu_{0}^{n}=P Q_{n}\right)$. Also under policy $\Pi^{n}$, let $\nu_{k}^{n}$ denote the distribution of $\left(X_{[0, k]}, Y_{[0, k]}\right)$ if the observation channel is $Q$ for all the stages $0, \ldots, t$. Then we have

$$
E_{P}^{Q^{n}, \Pi^{n}}\left[c\left(X_{t}, U_{t}\right)\right]=\int \mu_{0}^{n}\left(d x_{0}, d y_{0}\right) \zeta_{0, t}^{n}\left(x_{0}, y_{0}\right)
$$

and

$$
E_{P}^{Q, \Pi^{n}}\left[c\left(X_{t}, U_{t}\right)\right]=\int \nu_{t}^{n}\left(d x_{[0, t]}, d y_{[0, t]}\right) \zeta_{t, t}^{n}\left(x_{[0, t]}, y_{[0, t]}\right)
$$

Note that by construction, for all $k=1, \ldots, t$

$$
\begin{aligned}
& \int \mu_{k}^{n}\left(d x_{[0, k]}, d y_{[0, k]}\right) \zeta_{k, t}^{n}\left(x_{[0, k]}, y_{[0, k]}\right) \\
& =\int \nu_{k-1}^{n}\left(d x_{[0, k-1]}, d y_{[0, k-1]}\right) \zeta_{k-1, t}^{n}\left(x_{[0, k-1]}, y_{[0, k-1]}\right) .
\end{aligned}
$$

Thus each term in the sum on the right hand side of (6.2) can be expressed as a telescopic sum, which in turn can be bounded term-by-term, as follows:

$$
\begin{aligned}
\left|E_{P}^{Q^{n}, \Pi^{n}}\left[c\left(X_{t}, U_{t}\right)\right]-E_{P}^{Q, \Pi^{n}}\left[c\left(X_{t}, U_{t}\right)\right]\right|= & \mid \sum_{k=0}^{t} \int \mu_{k}^{n}\left(d x_{[0, k]}, d y_{[0, k]}\right) \zeta_{k, t}^{n}\left(x_{[0, k]}, y_{[0, k]}\right) \\
& -\int \nu_{k}^{n}\left(d x_{[0, k]}, d y_{[0, k]}\right) \zeta_{k, t}^{n}\left(x_{[0, k]}, y_{[0, k]}\right) \mid
\end{aligned}
$$




$$
\begin{aligned}
& \leq \sum_{k=1}^{t}\left\|\mu_{k}^{n}-\nu_{k}^{n}\right\|_{T V}\left\|\zeta_{k, t}^{n}\right\|_{\infty} \\
& \leq\|c\|_{\infty} \sum_{k=1}^{t}\left\|\mu_{k}^{n}-\nu_{k}^{n}\right\|_{T V} .
\end{aligned}
$$

For any Borel set $B \subset \mathbb{X}^{k} \times \mathbb{Y}^{k}$, define $B\left(x_{[0, k]}, y_{[0, k-1]}\right)=\left\{y_{k} \in \mathbb{Y}:\left(x_{[0, k]}, y_{[0, k]}\right) \in\right.$ $B\}$, so that

$$
\begin{aligned}
&\left|\mu_{k}^{n}(B)-\nu_{k}^{n}(B)\right|=\mid \int \nu_{k-1}^{n}\left(d x_{[0, k-1]}, d y_{[0, k-1]}\right) \int P\left(d x_{k} \mid x_{k-1}, \gamma_{k-1}^{n}\left(y_{[0, k-1]}\right)\right. \\
&\left(Q_{n}\left(B\left(x_{[0, k]}, y_{[0, k-1]}\right) \mid x_{k}\right)-Q\left(B\left(x_{[0, k]}, y_{[0, k-1]}\right) \mid x_{k}\right)\right) \mid \\
& \leq \sup _{x_{k} \in \mathbb{X}}\left\|Q_{n}\left(\cdot \mid x_{k}\right)-Q_{n}\left(\cdot \mid x_{k}\right)\right\|_{T V} .
\end{aligned}
$$

The preceding bound and the uniform convergence of $\left\{Q_{n}\right\}$ imply $\lim _{n}\left\|\mu_{k}^{n}-\nu_{k}^{n}\right\|_{T V}=$ 0 for all $k$. Combining this with (6.3) and (6.2) gives

$$
J\left(P, Q^{n}, \Pi^{n}\right)-J\left(P, Q, \Pi^{n}\right) \rightarrow 0 .
$$

Replacing $\Pi^{n}$ with $\Pi$ we can use an identical argument to show that $J\left(P, Q^{n}, \Pi\right) \rightarrow$ $J(P, Q, \Pi)$. Since $\epsilon>0$ in (6.1) was arbitrary, the proof is complete.

We obtained the continuity of the optimal cost on the space of channels equipped with a more stringent notion for convergence in total variation. This result and its proof indicate that further technical complications emerge in multi-stage problems. Likewise, upper semi-continuity under weak convergence and setwise convergence require more stringent uniformity assumptions, which we leave for future research.

One further interesting problem regarding the multi-stage case is to consider adaptive observation channels. For example, one may aim to design optimal adaptive quantizers for a control problem. In this case, Markov Decision Process tools can be used for obtaining existence conditions for optimal channels and quantizers. Some related results on optimal adaptive quantization are presented in [7].

7. Concluding remarks, some implications and future work. This paper studied the structural and topological properties of some optimization problems in stochastic control in the space of observation channels. The main problem we considered is how to approach appropriate notions of convergence and distance while studying communication channels in the context of stochastic control problems.

The restriction to Euclidean state spaces is not essential and many (but not all) of the positive results can be extended to the case where $\mathbb{X}, \mathbb{Y}$, and $\mathbb{U}$ are arbitrary Polish spaces. In particular, all the positive results in Sections 3 carry through without change, except Theorem 3.2. The results of Section 4 hold for this more general setup (however, in Lemma 4.3 we need the additional condition that the space is $\sigma$-compact). Likewise, most of the positive results in Section 5 on quantization hold more generally (in fact, Theorem 5.5 holds for an arbitrary measurable space), but two of the main results, Theorems 5.8 and 5.9 , do need the assumption that $\mathbb{X}$ is a finite-dimensional Euclidean space.

7.1. Sufficient conditions for continuity under setwise and weak convergence. A careful analysis of the proof of Theorem 3.4 reveals that we need a uniform convergence principle for setwise convergence to be sufficient for continuity. 
That is, we wish to have

$$
\lim _{n \rightarrow \infty} \sup _{\gamma \in \mathcal{F}}\left|\int\left(\int Q(d y \mid x) c(x, \gamma(y))-\int Q_{n}(d y \mid x) c(x, \gamma(y))\right) P(d x)\right|=0,
$$

where $\mathcal{F}$ is a set of allowable policies, to be able to have continuity under setwise convergence. Thus, one important question of practical interest, is the following: What type of stochastic control problems, cost functions, and allowable policies lead to solutions which admit such a uniform convergence principle under setwise convergence? Some sufficient conditions for uniform setwise convergence are presented in [30].

Likewise, a parallel discussion applies for weak convergence under the assumption that for every $Q_{n}$ and for $Q$, corresponding optimal policies $\gamma_{n}$ and $\gamma$ are continuous and are assumed to be from a given class of policies $\mathcal{F}$. One wants to have

$$
\int_{\mathbb{X} \times \mathbb{Y}} c\left(x, \gamma_{n}(y)\right) Q_{n}(d y \mid x) P(d x) \rightarrow \int_{\mathbb{X} \times \mathbb{Y}} c(x, \gamma(y)) Q(d y \mid x) P(d x) .
$$

A sufficient condition for this is the following form of uniform weak convergence:

$$
\lim _{n \rightarrow \infty} \sup _{\gamma \in \mathcal{F}}\left|\int_{\mathbb{X} \times \mathbb{Y}} c(x, \gamma(y)) Q_{n}(d y \mid x) P(d x)-\int_{\mathbb{X} \times \mathbb{Y}} c(x, \gamma(y)) Q(d y \mid x) P(d x)\right|=0 .
$$

7.2. Empirical consistency of optimal controllers. One issue to discuss is the connections of our results with consistency in learning the channel from empirical observations.

When one does not know the system dynamics, such as the observation channel, one typically attempts to learn the channel via test inputs or empirical observations. Let $\left\{\left(X_{i}, Y_{i}\right), i \in \mathbb{N}\right\}$ be an $\mathbb{X} \times \mathbb{Y}$-valued i.i.d sequence generated according to some distribution $\mu$. Define the the empirical occupation measures for every $n \in \mathbb{N}$, by letting

$$
\mu_{n}(B)=\frac{1}{n} \sum_{i=1}^{n} 1_{\left\{\left(X_{i}, Y_{i}\right) \in B\right\}},
$$

for every measurable $B \subset \mathbb{X} \times \mathbb{Y}$. Then one has $\mu_{n}(B) \rightarrow \mu(B)$ almost surely (a.s.) by the strong law of large numbers. However, it is generally not true that $\mu_{n} \rightarrow \mu$ setwise a.s. (e.g., $\mu_{n}$ never converges to $\mu$ setwise when either $X_{i}$ or $Y_{i}$ has a nonatomic distribution), in which case $\mu_{n}$ cannot converge to $\mu$ in total variation.

On the other hand, again by the strong law, for any $\mu$-integrable function $f$ on $\mathbb{X} \times \mathbb{Y}$, one has, almost surely,

$$
\lim _{n \rightarrow \infty} \int f(x, y) \mu_{n}(d x, d y)=\int f(x, y) \mu(d x, d y)
$$

In particular, $\mu_{n} \rightarrow \mu$ weakly with probability one [13].

In the learning theoretic context, the convergence of the costs optimal for $\mu_{n}$ to the cost optimal for $\mu$ is called the consistency of empirical risk minimization (see 32 . for an overview). In particular, if the cost function and the allowable control policies $\mathcal{F}$ are such that

$$
\lim _{n \rightarrow \infty} \sup _{\gamma \in \mathcal{F}}\left|\int c(x, \gamma(y)) \mu_{n}(d x, d y)-\int c(x, \gamma(y)) \mu(d x, d y)\right|=0,
$$


then we obtain consistency.

A class of measurable functions $\mathcal{E}$ is called a Glivenko-Cantelli class [12, if the integrals with respect to the empirical measures converge almost surely to the integrals with respect to the true measure uniformly over $\mathcal{E}$. Thus, if

$$
\mathcal{G}=\{\gamma: c(x, \gamma(y)) \in \mathcal{E}\},
$$

where $\mathcal{E}$ is a class of Glivenko-Cantelli family of functions, then we could establish consistency. One example of a Glivenko-Cantelli family of real functions on $\mathbb{R}^{N}$ is the family $\left\{f:\|f\|_{B L} \leq M\right\}$ for some $0<M<\infty$, where $\|\cdot\|_{B L}$ denotes the bounded Lipschitz norm [12].

Thus, if we restrict the class of control policies, and given a cost function, we can obtain consistency and robustness to mismatch in the channel due to learning. The classification of the class of objective functions and policies which would lead to such a consistency result is a future research problem.

\section{Appendix.}

8.1. Proof of Lemma 2.2. (i) Since $c(x, \cdot)$ is continuous and bounded on $\mathbb{Y}$ for all $x$, we have

$$
\begin{aligned}
\lim _{n \rightarrow \infty} \int_{\mathbb{X} \times \mathbb{Y}} c(x, y) P Q_{n}(d x d y) & =\lim _{n \rightarrow \infty} \int_{\mathbb{X}}\left(\int_{\mathbb{Y}} c(x, y) Q_{n}(d y \mid x)\right) P(d x) \\
& =\int_{\mathbb{X}}\left(\int_{\mathbb{Y}} c(x, y) Q(d y \mid x)\right) P(d x) \\
& =\int_{\mathbb{X} \times \mathbb{Y}} c(x, y) P Q(d x, d y)
\end{aligned}
$$

where first we used Fubini's theorem, and then the dominated convergence theorem and the fact that $\int_{\mathbb{X}} c(x, y) Q_{n}(d y \mid x)$ is bounded and converges to $\int_{\mathbb{X}} c(x, y) Q(d y \mid x)$ for $P$-a.e. $x$.

(ii) Let $A \in \mathcal{B}(\mathbb{X} \times \mathbb{Y})$ and for $x$, let $A_{x}=\{y:(x, y) \in A\}$. Similarly to the previous proof,

$$
\begin{aligned}
\lim _{n \rightarrow \infty} P Q_{n}(A) & =\lim _{n \rightarrow \infty} \int_{\mathbb{X}} Q_{n}\left(A_{x} \mid x\right) P(d x) \\
& =\int_{\mathbb{X}} Q\left(A_{x} \mid x\right) P(d x) \\
& =P Q(A)
\end{aligned}
$$

by the dominated convergence theorem since $\lim _{n \rightarrow \infty} Q_{n}\left(A_{x} \mid x\right)=Q\left(A_{x} \mid x\right)$ for $P$-a.e. $x$.

(iii) We have

$$
\begin{aligned}
\sup _{A \in \mathcal{B}(\mathbb{X} \times \mathbb{Y})}\left|P Q_{n}(A)-P Q(A)\right| & =\sup _{A \in \mathcal{B}(\mathbb{X} \times \mathbb{Y})}\left|\int_{\mathbb{X}} Q_{n}\left(A_{x} \mid x\right) P(d x)-\int_{\mathbb{X}} Q\left(A_{x} \mid x\right) P(d x)\right| \\
& \leq \sup _{A \in \mathcal{B}(\mathbb{X} \times \mathbb{Y})} \int_{\mathbb{X}}\left|Q_{n}\left(A_{x} \mid x\right)-Q\left(A_{x} \mid x\right)\right| P(d x) \\
& \leq \int_{\mathbb{X}} \sup _{B \in \mathcal{B}(\mathbb{Y})}\left|Q_{n}(B \mid x)-Q(B \mid x)\right| P(d x) .
\end{aligned}
$$


Since $\sup _{B \in \mathcal{B}(\mathbb{Y})}\left|Q_{n}(B \mid x)-Q(B \mid x)\right| \rightarrow 0$ for $P$-a.e. $x$, an application of the dominated convergence theorem completes the proof.

8.2. Proof of Theorem 3.1. We have

$$
J(P, Q)=\inf _{\gamma \in \mathcal{G}} \int_{\mathbb{X} \times \mathbb{Y}} c(x, \gamma(y)) Q(d y \mid y) P(d x) .
$$

Let $(X, Y) \sim P Q$ and let $P(\cdot \mid y)$ be the (regular) conditional distribution of $X$ given $Y=y$. If $(P Q)_{\mathbb{Y}}$ denotes the distribution of $Y$, then

$$
\begin{aligned}
J(P, Q) & =\inf _{\gamma \in \mathcal{G}} \int_{\mathbb{Y}} \int_{\mathbb{X}} c(x, \gamma(y)) P(d x \mid y)(P Q)_{\mathbb{Y}}(d y) \\
& =\int_{\mathbb{Y}}\left(\inf _{u \in \mathbb{U}} \int_{\mathbb{X}} c(x, u) P(d x \mid y)\right)(P Q)_{\mathbb{Y}}(d y) .
\end{aligned}
$$

where the validity of the second equality is explained below.

By assumption A3, $c$ is bounded and $c\left(x, u_{n}\right) \rightarrow c(x, u)$ if $u_{n} \rightarrow u$ for all $x$; thus by the dominated convergence theorem

$$
\int_{\mathbb{X}} c\left(x, u_{n}\right) P(d x \mid y) \rightarrow \int_{\mathbb{X}} c(x, u) P(d x \mid y)
$$

proving that $g(u, y)=\int_{\mathbb{X}} c(x, u) P(d x \mid y)$ is continuous in $u$ for each $y$. Since $\mathbb{U}$ is compact, there exists $\gamma^{*}(y) \in \mathbb{U}$ such that $g\left(\gamma^{*}(y), y\right)=\inf _{u \in \mathbb{U}} g(u, y)$. A standard argument shows that $\gamma^{*}: \mathbb{Y} \rightarrow \mathbb{U}$ can be taken to be measurable (see, e.g., Appendix D of [19]) and we have

$$
J(P, Q)=\int_{\mathbb{X} \times \mathbb{Y}} c\left(x, \gamma^{*}(y)\right) Q(d y \mid y) P(d x) .
$$

\section{REFERENCES}

[1] E. A. Abaya and G. L. Wise, "Convergence of vector quantizers with applications to optimal quantization, SIAM Journal on Applied Mathematics, vol. 44, pp. 183-189, 1984.

[2] Y. Bar-Shalom and E. Tse, "Dual effect, certainty equivalence and separation in stochastic control", IEEE Transactions on Automatic Control, vol.19, pp. 494-500, October 1974.

[3] A. Barvinok, A Course in Convexity, vol. 54 of Graduate Studies in Mathematics. Providence, RI: American Mathematical Society, 2002.

[4] P. Billingsley, Convergence of Probability Measures. New York: Wiley 1968.

[5] P. Billingsley, Probability and Measure. New York: Wiley, 2nd ed., 1986.

[6] V. I. Bogachev, Measure Theory. Berlin, Heidelberg: Springer Verlag, 2007.

[7] V. S. Borkar, S. K. Mitter, and S. Tatikonda, "Optimal sequential vector quantization of Markov sources," SIAM Journal on Control and Optimization, vol. 40, pp. 135-148, 2001.

[8] C.D. Charalambous and F. Rezaei, "Stochastic uncertain systems subject to relative entropy constraints: Induced norms and monotonicity properties of minimax games", IEEE Transactions on Automatic Control, vol. 52, no. 4, pp 647-663, May 2007.

[9] T. M. Cover and J. A. Thomas, Elements of Information Theory, Wiley, NY, 1991.

[10] L. Devroye, and L. Györfi, Non-parametric Density Estimation: The L 1 View, New York: John Wiley, 1985.

[11] L. Devroye and L. Györfi "No empirical measure can converge in the total variation sense for all distributions," Annals of Statistics, 18, pp. 1496-1499, 1990.

[12] R.M. Dudley, E. Gine, and J. Zinn, "Uniform and universal Glivenko-Cantelli classes", Journal of Theoretical Probability, vol. 4, pp. 485-510, 1991.

[13] R. M. Dudley, Real Analysis and Probability, Cambridge University Press, Cambridge, 2nd ed., 2002. 
[14] J. K. Ghosh, and R. V. Ramamoorthi, Bayesian Nonparametrics, Springer, New York, 2003.

[15] S. Graf and H. Luschgy, Foundations of Quantization for Probability Distributions. Berlin, Heidelberg: Springer Verlag, 2000.

[16] R. M. Gray and L. D. Davisson, "Quantizer mismatch," IEEE Transactions on Communications, vol. 23, pp. 439-443, 1975.

[17] L. Györfi and M. Kohler, "Nonparametric estimation of conditional distributions," IEEE Transactions on Information Theory, vol. 53, pp. 1872-1879, May 2007.

[18] A. György and T. Linder, "Codecell convexity in optimal entropy-constrained vector quantization," IEEE Transactions on Information Theory, vol. 49, pp. 1821-1828, July 2003.

[19] O. Hernandez-Lerma, J.B. Lasserre, Discrete-Time Markov Control Processes, Basic Optimality Criteria, Springer-Verlag, New York, 1996.

[20] O. Hernandez-Lerma, J.B. Lasserre, Markov Chains and Invariant Probabilities, BirkhäuserVerlag, Basel, 2003.

[21] P. J. Huber, "Robust estimation of a location parameter", The Annals of Mathematical Statistics, vol. 35, No. 1 (Mar., 1964), pp. 73-101.

[22] T. Linder, "On the training distortion of vector quantizers", IEEE Transactions on Information Theory, vol. 46, pp. 1617-1623, 2000.

[23] N. Muraki and M. Ohya, "Note on continuity of information rate," Illinois Journal of Mathematics, vol. 36, pp. 529-550, Winter 1992.

[24] D. Pollard, "Quantization and the method of $k$-means, IEEE Transactions on Information Theory, vol. 28, pp. 199-205, 1982.

[25] H. V. Poor, "On robust Wiener filtering, IEEE Transactions on Automatic Control, vol. AC-25, pp. 521-526, Jun. 1980.

[26] F. Rezaei, C.D. Charalambous and N. U. Ahmed, "Optimization of stochastic uncertain systems with variational norm constraints", in Proc. IEEE Conference on Decision and Control, pp. 2159-2163, New Orleans, LA, USA, Dec. 2007

[27] W. Rudin, Real and Complex Analysis, New York: McGraw-Hill, 3rd ed., 1987.

[28] Y. Socratous, F. Rezaei and C.D. Charalambous, "Nonlinear estimation for a class of systems", IEEE Transactions on Information Theory, vol. 55, no. 4, pp. 1930-01938, Apr. 2009.

[29] J. N. Tsitsiklis, "Extremal properties of likelihood-ratio quantizers", IEEE Transactions on Communications, Vol. 41, pp. 550-558, Apr. 1993.

[30] F. Topsoe, "Uniformity in convergence of measures", Z. Wahrscheinlichkeitsth, vol. 39, pp. $1-30,1977$.

[31] R. L. Wheeden and A. Zygmund, Measure and Integral, New York: Marcel Dekker, 1977.

[32] V. N. Vapnik The Nature of Statistical Learning Theory Springer, New York, 2nd ed., 2000.

[33] H. S. Witsenhausen, "On the structure of real-time source coders," Bell Syst. Tech. J., 58:14371451, July/August 1979.

[34] Y. Wu and S. Verdú, "Functional properties of MMSE", Proc. IEEE International Symposium on Information Theory, Austin, TX, June 13-18, 2010. 